\title{
Telomere dysfunction promotes small vessel vasculitis via the LL37-NETs-dependent mechanism
}

\author{
Yingying Lu ${ }^{1,2,3,4 \#}$, Hong Jiang ${ }^{1,2,3,4 \#}$, Bingjue $\mathrm{Li}^{1,2,3,4}$, Luxi Cao ${ }^{1,2,3,4}$, Qixia Shen ${ }^{1,2,3,4}$, Weiwei Yi ${ }^{5}$, Zhenyu Ju ${ }^{5}$, \\ Liangliang Chen ${ }^{1,2,3,4}$, Fei Han ${ }^{1,2,3,4}$, Daniel Appelgren ${ }^{6}$, Mårten Segelmark ${ }^{6}$, Nicole de Buhr ${ }^{7,8}$, Maren von \\ Köckritz-Blickwede ${ }^{7,8}$, Jianghua Chen ${ }^{1,2,3,4}$
}

${ }^{1}$ Kidney Disease Center, The First Affiliated Hospital, College of Medicine, Zhejiang University, Hangzhou 310003, China; ${ }^{2}$ Kidney Disease Immunology Laboratory, The Third Grade Laboratory, State Administration of Traditional Chinese Medicine of PR China, Hangzhou 310003, China; ${ }^{3}$ Key Laboratory of Multiple Organ Transplantation, Ministry of Health, Hangzhou 310003, China; ${ }^{4}$ Key Laboratory of Nephropathy, Hangzhou 310003, China; ${ }^{5}$ Institute of Aging Research and Max-Planck-Research Group on Stem Cell Aging, Hangzhou Normal University, Hangzhou 311121, China; ${ }^{6}$ Department of Medical and Health Sciences (IMH), Linkoping University, Linkoping, Sweden; ${ }^{7}$ Department of Physiological Chemistry, University of Veterinary Medicine Hannover, Hannover, Germany; ${ }^{8}$ Department of Physiological Chemistry and Research Center for Emerging Infections and Zoonoses (RIZ), University of Veterinary Medicine Hannover, Hannover, Germany

Contributions: (I) Conception and design: H Jiang, J Chen; (II) Administrative support: J Chen, Z Ju, M Segelmark, MV Köckritz-Blickwede; (III) Provision of study materials or patients: L Chen, F Han; (IV) Collection and assembly of data: Y Lu, H Jiang, B Li, L Cao, Q Shen, W Yi, D Appelgren, ND Buhr, MV Köckritz-Blickwede; (V) Data analysis and interpretation: Y Lu, H Jiang; (VI) Manuscript writing: All authors; (VII) Final approval of manuscript: All authors.

"These authors contributed equally to this work.

Correspondence to: Professor Hong Jiang, PhD; Professor Jianghua Chen, MD. Kidney Disease Center, The First Affiliated Hospital, School of Medicine, Zhejiang University, Hangzhou 310003, China. Email: annie.jh@163.com; chenjianghua@zju.edu.cn.

Background: Small vessel vasculitis (SVV) is a group of systemic autoimmune diseases that are mediated by neutrophil extracellular traps (NETs) in response to cathelicidin LL37, an aging molecular marker, which could be induced by telomere dysfunction. Therefore, in this study, we evaluated the hypothesis that telomere dysfunction in neutrophils may promote SVV via an LL37-NETs-dependent mechanism.

Methods: We contrasted the release of neutrophil NETs from mice with telomere dysfunction, mice with DNA damage and wide-type mice. Neutrophil telomere length, the expression of LL37, and the formation of NETs were measured in SVV patients and healthy controls (HCs). The co-expression of $\gamma \mathrm{H} 2 \mathrm{AX}, \mathrm{LL} 37$, and NETs were detected in SVV patients to evaluate the association of the immune aging of neutrophils and pro-inflammatory conditions. LL37 inhibitor was used to verify its key role in NETs release in SVV patients and DNA damage mice.

Results: We found that NETs were over-induced by telomere dysfunction and DNA damage in mice, which may be associated with a marked increase in LL37. For patients with SVV, telomeres in neutrophils were significantly shortened, which was also associated with higher levels of LL37 and NETs. Inhibition of LL37 reduced the NETs released from neutrophils.

Conclusions: Taken together, the results of these studies suggest that dysfunction of telomeres may promote SVV through the mechanism of LL37-dependent NETs. Thus, targeting the LL37-NETs may be a novel therapy for SVV.

Keywords: Telomere dysfunction; DNA damage; small vessel vasculitis (SVV); LL37; neutrophil extracellular traps (NETs)

Submitted Oct 07, 2019. Accepted for publication Jan 30, 2020.

doi: 10.21037/atm.2020.02.130

View this article at: http://dx.doi.org/10.21037/atm.2020.02.130 


\section{Introduction}

Small vessel vasculitis (SVV) is a group of systemic autoimmune diseases that are characterized by necrotizing inflammation within the vessel wall and the presence of anti-neutrophil cytoplasmic autoantibody (ANCA) (1). This happens mostly in the elderly, with a peak age of 65 to 74 years (2). When the kidney is involved, it manifests as pauci-immune necrotizing and crescentic glomerulonephritis pathophysiologically (2). Neutrophils play a key role in the pathogenic process since they infiltrate the renal interstitium, necrotizing capillary loops, and the crescent of glomerulus during the acute stage. These are both effector cells and autoimmune vectors for the disease $(3,4)$.

Recent studies implicated the role of neutrophil extracellular traps (NETs) in breaking immune tolerance and promoting organ damage in SVV $(5,6)$. NETs are characterized by the presence of neutrophil nuclear DNA fibers in the extracellular space. Histones and antimicrobial proteins such as myeloperoxidase (MPO), proteinase- 3 (PR3), and human cathelicidin LL37 reside on them (7). Previous studies found that neutrophils activated by ANCA produced NETs and released LL37 in vitro (8). There was also evidence for NETs formation and LL37 deposition in kidney tissue in individuals with SVV (8). Interestingly, in 2014, Neumann et al. found the role of LL37 in facilitating the formation and stabilization of the structure of NETs $(9,10)$. The interaction of LL37 and NETs may be a new mechanism for SVV.

We reported a group of biomarkers for aging induced by telomere dysfunction and DNA damage in 2008 (11). These markers were not only highly expressed in old, fourth generations of telomerase knockout mice (G4 mTerc ${ }^{-1}$ ) but were also increasingly expressed during human aging and in telomere-dysfunction-related diseases, such as cirrhosis and myelodysplastic syndromes (MDS) (11).

LL37 related to NETs seems pathogenic for SVV. However, whether LL37 induced by telomere-dysfunction has a role in regulating NETs in SVV is still unknown.

Hence, we proposed that the pathological immune senescence of aging neutrophils will negatively affect their activity due to DNA damage and telomere dysfunction. As a result, it would develop more LL37 and interfere with NETs, ultimately leading to renal damage and SVV progression.

\section{Methods}

\section{Animals}

$\mathrm{Terc}^{+/}$mice were provided by the Institute of Aging Research and Max-Planck-Research Group on Stem Cell Aging, Hangzhou Normal University. In order to obtain the fourth generation of telomerase knockout (G4mTerc ${ }^{-1}$ ) mice with severe telomere dysfunction, $\mathrm{Terc}^{+/}$mice were intercrossed to produce $\mathrm{Terc}^{-/-}$mice, and then $\mathrm{Terc}^{-/-}$mice were intercrossed successively to decrease telomere reserves in the pathogen-free animal facility of Zhejiang University. All mice were of a C57BL/6J background. The experiments were approved by our institution's Ethics Committee for Investigation with Animals.

\section{The isolation of neutrophils and irradiation procedures used}

Murine neutrophils were isolated from bone marrow, as previously described (10). Briefly, bone marrow cells were flushed from the femur and tibia with RPMI-1640 (life technologies) $+2 \%$ Fetal Bovine Serum (FBS, Life technologies). After filtration through a cell strainer (100 $\mu \mathrm{m}$; BD Falcon, San Jose, CA, USA) and erythrocyte lysis with sterile water, the cells were incubated for $1 \mathrm{~h}$ at $37{ }^{\circ} \mathrm{C}$ with $5 \% \mathrm{CO}_{2}$ to separate the suspended granulocytes from adherent monocytes.

PolymorphPrep ${ }^{\mathrm{TM}}$ (Progen Biotechnik) was used according to the manufacturer's instructions, as previously described (9), to isolate human neutrophils from fresh blood.

For the irradiation assay, neutrophils were irradiated with a dose of 10 Gy using an RS-2000 X-Ray Biological Irradiator (Rad Source Technologies) after being seeded onto 24-well plates.

\section{Visualization of NETs formed in vitro}

In vitro, NETs were visualized as previously described $(9,10)$. Briefly, a total of $5 \times 10^{5}$ neutrophils/well were seeded in 24-well plates and cultivated in the presence or absence of $40 \mu \mathrm{g} / \mathrm{mL}$ aprotinin (A1153 Sigma) at $37{ }^{\circ} \mathrm{C}$ in $5 \% \mathrm{CO}_{2}$ for 3 hours. After fixation, permeabilization, and blocking, cells were incubated with mouse anti-DNA/histone 1 monoclonal antibody (MAB 38624, Millipore, USA), followed by Alexa-Fluor-488-labeled goat-anti-mouse 
Table 1 The characteristics of human samples

\begin{tabular}{|c|c|c|c|}
\hline Characteristics & $\operatorname{SVV}(n=70)$ & $\mathrm{HCs}(\mathrm{n}=70)$ & $P$ value \\
\hline Age, mean $\pm S D$, year & $56.46 \pm 13.51$ & $56.40 \pm 5.17$ & 0.9737 \\
\hline WBC, mean $\pm S D, n \times 10^{9}$ & $7.85 \pm 3.43$ & $5.62 \pm 1.72$ & $<0.0001$ \\
\hline ANCA at diagnosis, PR3-ANCA/MPO-ANCA/ANCA negative, $n$ & $63 / 4 / 3$ & NA & NA \\
\hline Estimated GFR, medium (IQR), mL/minute/1.73 $\mathrm{m}^{2}$ & $20(9.025-36.70)$ & $109.675(96.59-121.54)$ & $<0.0001$ \\
\hline Urinary protein, mean $\pm S D, g / 24$ hours & $2.34 \pm 1.647$ & NA & NA \\
\hline
\end{tabular}

WBC, white blood cell count; IQR, interquartile range; eGFR, estimated glomerular filtration rate; NA, not available.

antibody (Life Technologies). Slides were mounted, and images were randomly acquired using confocal fluorescence microscopy installed with a Leica TCS SP8 microscope with an HCX PL APO CS2 $63 \times$ oil immersion objective. Control preparations treated with an isotype antibody was used to adjust the settings. For each preparation, a minimum of five random images from three independent subjects was acquired. Data were expressed as percentages of NET-producing neutrophils.

\section{Subjects}

The study protocols followed the Declaration of Helsinki. SVV was diagnosed according to the Chapel Hill Consensus Conference (CHC) definitions (12). SVV patients with renal involvement $(\mathrm{n}=70)$ and kidney transplantation donors, namely healthy controls (HCs, $n=70$ ), were recruited from our hospital with written consent. There was no significant difference in the age between the two groups $(56.46 \pm 1.615$ vs. $56.40 \pm 0.6173, \mathrm{P}=0.9737)$. The ratio of males and females was the same between the two groups (male/female $=25 / 45$ ), thereby avoiding the potential effects of sexual differences. The sample characteristics are presented in Table 1. Blood samples and specimens from renal needle biopsies were obtained and used anonymously.

\section{Real-time polymerase chain reaction (RT-PCR)}

We used RT-PCR to determine the telomere length of human neutrophils. Firstly, genomic DNA (Axygen, USA) was extracted from human neutrophils, and the telomere length of a set of standard cell lines was assessed by Southern blotting. Then, RT-PCR was carried as previously described (13). Briefly, Telomere (T) PCR and single-copy gene human $\beta$-globin (S) PCR were conducted. 126 The reactions were performed on a Prism7500 (Applied 127 Biosystems), and data were collected and analyzed using 128 ABI Prism7500 SDS v.1.7 software. 129

\section{Indirect Enzyme-Linked Immunosorbent Assay (ELISA)} of $L L 37$

After coating the microwell plates (NUNC) with $50 \mu \mathrm{L}$ serum, we applied a $1 \%$ milk-Phosphate Buffered Saline (PBS) solution to block the added binding sites on the plastic surface. Then, the plates were incubated with the primary antibody, mouse-anti-human LL-37 (Hycult biotech, The Netherlands) 1:500 (v/v) and horseradish peroxidase (HRP)-hapten conjugated secondary antibody (DW0990, Dawen Biotec, USA) 1:2,000 (v/v) at room temperature for $2 \mathrm{~h}$. After each step, the plates were washed three times with PBST wash solution (PBS with $0.05 \%$ Tween 20) to remove the unattached material. Each well was then incubated with $100 \mu \mathrm{L}$ tetramethyl benzidine peroxide-based substrate solution for $15 \mathrm{~min}$. The color reaction was stopped with $20 \mu \mathrm{L} /$ well $2 \mathrm{M} \mathrm{H}_{2} \mathrm{SO}_{4}$ stop solution. Absorbances were at once read in the microplate reader at $450 \mathrm{~nm}$ (ELX800NB BioTek USA).

\section{Cell-free (cf)-DNA}

To quantify levels of free DNA released into circulation in human serum samples, the Quant-iT Pico Green doublestranded DNA (dsDNA) assay was conducted according to the manufacturer's instructions (Invitrogen, Germany). It is based on an ultrasensitive fluorescent nucleic acid stain of dsDNA in solution. The serum samples were diluted 10-fold with TE buffer (10 mM Tris-HCl, $1 \mathrm{mM}$ EDTA, 
$\mathrm{pH}$ 7.5) before detection. A standard calibration curve was acquired using a lambda DNA standard in all analyses. The fluorescence intensity was measured with a fluorescence microplate reader (excitation at $480 \mathrm{~nm}$ wavelength, emission at $520 \mathrm{~nm}$ wavelength, infinite M200, TECAN) and quantified using Magellan V6.1 software.

\section{NETs remnant ELISA}

NETs remnant ELISA was conducted as previously described (14). Briefly, after coating 96-well microplates with a monoclonal mouse anti-nucleosome antibody (B6. SLE-1, $0.5 \mathrm{mg} / \mathrm{mL}$ ) and blocking the additional binding sites, the plates were incubated with standards and serum for $2 \mathrm{~h}$ at room temperature. A rabbit anti-human MPO (1:500; DAKO, Carpinteria, CA, USA) was then added for $1 \mathrm{~h}$ at room temperature and an alkaline phosphataseconjugated swine anti-rabbit antibody (1:500, DAKO) for another hour. Finally, a substrate for alkaline phosphatase (4-nitrophenyl phosphate disodium salt hexahydrate; Sigma-Aldrich, St Louis, MO, USA) was added, and the plates were read at $405 \mathrm{~nm}$ using a VersaMax ELISA microplate reader (Molecular Devices, Sunnyvale, CA, USA). A standard curve was constructed for each plate, and all samples were interpolated from it.

\section{Immunofluorescent staining of in situ NETs}

Immunofluorescent staining was performed on frozen sections of kidney needle biopsies to obtain in situ evidence of NET formation. After permeabilization with $0.2 \%$ Triton X-100 and blocking with $1.5 \%$ goat serum, slides were incubated with a monoclonal antibody, mouse antiDNA/histone 1 (MAB 38624, Millipore, USA), followed by a secondary antibody, Alexa-Fluor-488-labeled goat-antimouse antibody (Life Technologies). For the co-localization assay of NETs and LL37, rabbit-anti-human LL-37 and Alexa-Fluor-594-labeled goat-anti-rabbit antibody were co-incubated with the corresponding antibody. Slides were mounted in ProlongGold ${ }^{\circledR}$ antifade with DAPI (136933; Invitrogen) and viewed using confocal fluorescence microscopy with a Leica TCS SP5 microscope with an HCX PL APO 40x oil immersion objective.

\section{Telomere, $r H 2 A X$, and LL37 triple-staining}

Briefly, after paraffin-embedded sections from kidney needle biopsies were dewaxed and the antigens were retrieved, the 207 slides were incubated in acidified pepsin (Sigma Aldrich, 208 USA) and dehydrated through graded alcohols. A FITC- 209 labeled PNA probe specific for (TTAGGG)n sequences 210 (PANAGENE, Korea) was co-denatured with the slide at 211 $80{ }^{\circ} \mathrm{C}$ for $3 \mathrm{~min}$ and hybridized at RT for $2 \mathrm{~h}$. Slides were 212 then incubated first with the mouse-anti-human LL37 213 (HM2070, Hycult biotech, The Netherlands) and rabbit- 214 anti- $\gamma \mathrm{H} 2 \mathrm{AX}$ (MABE205, Millipore, USA) antibodies, 215 followed by the secondary Alexa-Fluor-594-labeled goat- 216 anti-mouse antibody and Alexa-Fluor-488-labeled goat- 217 anti-rabbit antibody. Slides were mounted ProlongGold ${ }^{\circledR} \quad 218$ antifade with DAPI (Invitrogen, Paisley, UK) and finally 219 visualized with confocal fluorescence microscopy using a 220 Leica TCS SP8 microscope with an HCX PL APO CS2 221 $63 \times$ oil immersion objective.

\section{Statistical analyses}

Statistical analyses were performed using the MedCalc software package (MedCalc for Windows 8.1.1.0, MedCalc Software), SPSS16 .0, (SPSS, Inc., Chicago, IL, USA) and GraphPad Prism 5 (San Diego, CA, USA). A one-sample Kolmogorov-Smirnov test was performed to evaluate the normal distribution of data. The unpaired Student's $t$-test or Mann-Whitney test was used for the assessment of statistical significance between two groups with continuous variables. The results are presented as the mean \pm SEM or the mean \pm SD or the median (interquartile range). The error bars are present in all figures. Data were considered statistically significant at $\mathrm{P}<0.05$.

\section{Results}

NETS are over-induced in response to telomere dysfunction and DNA damage in mice

The fourth generation of telomerase knockout (G4mTerc ${ }^{-/}$) mice exhibited aging condition when they were 12 months old, while wide type mice (WT) did not start until 24 months old (15). So, we identified NETs released by the neutrophils from 12-month-old G4mTerc ${ }^{-/-}$mice (as a premature aging group), 12-month-old WT (as same-age control), and 24-month-old WT (as same aging condition control). The percentage of netting neutrophils increased in 12-month-old G4mTerc ${ }^{-/-}$mice compared to 12-monthold WT (mean \pm SEM: $24.69 \% \pm 2.32 \%$ vs. $12.06 \% \pm 0.72 \%$, $\mathrm{P}<0.0001$, Figure $1 A$ ) or 24 -month-old WT (mean $\pm \mathrm{SEM}$ : 
A
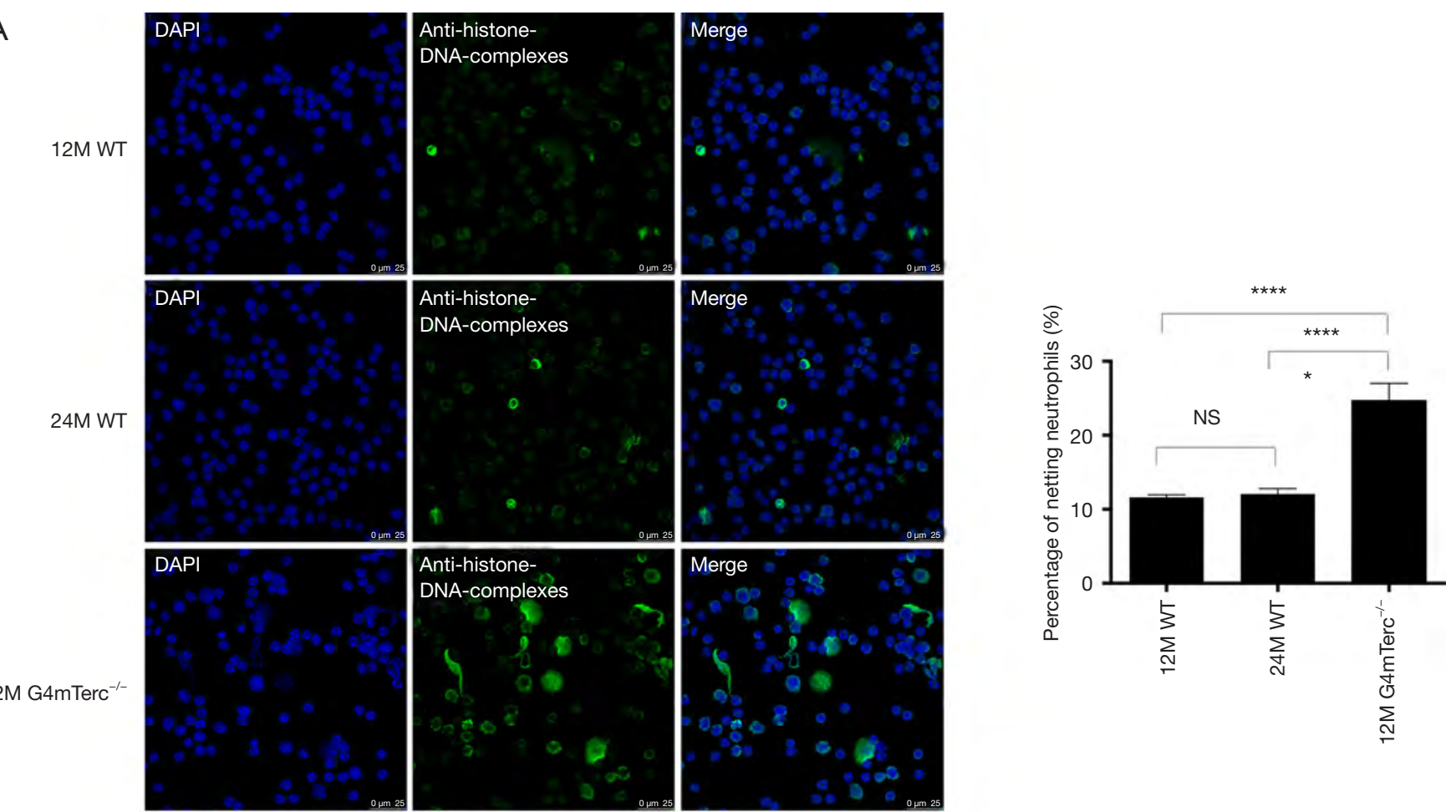

B
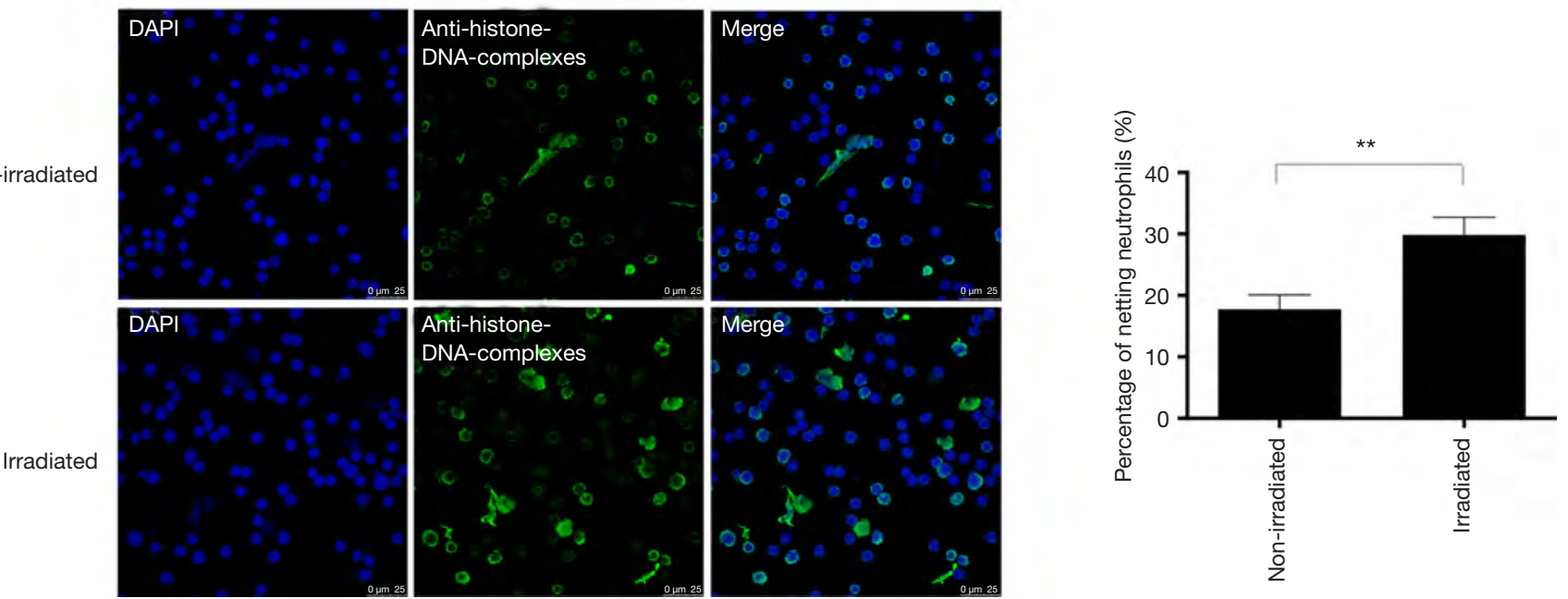

Figure 1 Mice neutrophils produce more NETs in response to telomere dysfunction or DNA damage. (A) The percentage of netting neutrophils from the 12-month-old fourth generations of telomerase knockout mice (G4mTerc $\left.{ }^{-1}\right)$ and wide type mice of different ages (WT); (B) the netting rate of irradiated and non-irradiated neutrophils from 2-month-old WT mice. The graph is a minimum of 15 images derived from 3 independent experiments. The error bars of the graph represent SEM. Unpaired Student's $t$-test was conducted to analyze the statistical significance of two independent experiments. ****, $\mathrm{P}<0.0001$; **, $\mathrm{P}<0.01$, NS, not significant; $2 \mathrm{M}, 2$-month-old; $12 \mathrm{M}, 12$-monthold; 24M, 24-month-old. 
Compared to non-irradiated neutrophils, irradiated neutrophils that suffered DNA damage tended to form more NETs (mean \pm SEM: $29.86 \% \pm 2.85 \%$ vs. $17.76 \% \pm 2.33 \%$, $\mathrm{P}=0.0027$, Figure $1 B)$.

\section{Neutrophils from SVV patients have shortened telomeres and produce higher levels of LL37 and NETs in vivo}

We used real-time polymerase chain reaction to detect the telomere lengths of neutrophils from SVV patients and HCs. The telomeres of neutrophils from SVV patients were significantly shortened compared to those from healthy individuals (mean \pm SEM: $8.35 \pm 0.24 v s$. $11.32 \pm 0.26$, $\mathrm{P}<0.0001$, Figure $2 A$ ).

The relative concentration of LL37 was measured using enzyme-linked immunosorbent assay (ELISA). The results were expressed as the optical density value. We found significantly increased serum LL37 levels in patients with SVV compared to HCs (mean \pm SEM: $1.229 \pm 0.024 v s$. $0.954 \pm 0.014$ a.u., $\mathrm{P}<0.0001$, Figure $2 B$ ). The concentration of urine LL37 was low in both groups. However, urine LL37 levels in patients with SVV remained higher than in HCs (mean \pm SEM: $0.032 \pm 0.005$ vs. $0.009 \pm 0.001$ a.u., $\mathrm{P}<0.0001$, Figure 2C).

We quantified NETs in circulation by evaluating the levels of major NET components, a NET remnant (nucleosome-MPO complexes), and cell-free DNA (cf-DNA).

Increased levels of NET remnants were found in patients with SVV compared to HCs (mean \pm SEM: 252.6 \pm 25.83 vs. $184.3 \pm 12.61$ a.u., $\mathrm{P}=0.0189$ ) (Figure $2 D$ ). Patients with SVV also showed higher cf-DNA levels compared with healthy individuals (mean \pm SEM: $393.9 \pm 19.78$ vs. $269.2 \pm$ $8.552 \mathrm{ng} / \mathrm{mL} ; \mathrm{P}<0.0001$, Figure $2 E)$.

Immunofluorescent staining showed that NETs were deposited in the kidney tissue of SVV patients, while no NET deposition was found in HCs (Figure $2 F$ ). Furthermore, in situ, NETs were decorated with the immunostimulatory peptide LL37 (Figure 2G).

\section{Aging neutrophils with telomere shortening and DNA damage produce LL37 and NETs in the renal injury of SVV patients}

To evaluate the association of the immune aging of neutrophils and pro-inflammatory conditions, we conducted telomere- $\gamma \mathrm{H} 2 \mathrm{AX}$, a double-stranded DNA damage marker, and LL37 triple-staining (Figure 3A). As shown in Figure 3A, neutrophils, characterized by containing a polymorphic 309 nucleus, infiltrated the kidney tissue of SVV patients. In 310 these polymorphic nuclei, the staining of $\gamma \mathrm{H} 2 \mathrm{AX}$ was co- 311 localized to telomere staining, which showed telomere 312 damage. During this time, LL37 assembled at the nuclear 313 periphery. We also captured a field of LL37 surrounding a 314 polymorphic nucleus in the background of NET deposition 315 in the kidney tissue of SVV patients (Figure 3B). These 316 results show that the immune aging of neutrophils and 317 inflammatory medium release co-exist in SVV patients. And 318 LL37 may build a bridge between telomere dysfunction and 319 NETs production of neutrophils. 320

\section{Neutrophils from SVV produce more NETs in vitro, and LL37 mediates this process}

To investigate whether neutrophils from SVV were more active in the circulation, we evaluated the ability for NET production by neutrophils from HCs and SVV patients in vitro. As shown in Figure 4A, Neutrophils from SVV patients produced more NETs than those from HCs (mean \pm SEM: $54.66 \% \pm 5.12 \%$ vs. $33.18 \% \pm 2.70 \%, \mathrm{P}=0.0002$, Figure $4 A$ ).

We also conducted an irradiation assay to induce DNA damage in neutrophils from healthy donors. Irradiated human neutrophils tended to produce more NETs compared to non-irradiated human neutrophils (mean \pm SEM: $47.48 \% \pm 2.77 \%$ vs. $33.18 \% \pm 2.70 \%, \mathrm{P}=0.0018$, Figure $4 A$ ). There was no significant difference in NET production ability between SVV neutrophils or irradiated neutrophils ( $\mathrm{P}=0.2276$, Figure $4 A)$.

Interestingly, when SVV neutrophils were treated with aprotinin, a serine protease inhibitor, to competitively block the activation of endogenous LL-37 (16), a significantly lower degree of NET formation was detectable (mean \pm SEM: $29.79 \% \pm 3.92 \%$ vs. $50.25 \% \pm 4.71 \%, \mathrm{P}=0.0024$, Figure $4 B$ ). However, aprotinin did not affect the NET release of neutrophils from healthy subjects (mean \pm SEM: $29.59 \% \pm 4.03 \%$ vs. $30.74 \% \pm 3.77 \%, \mathrm{P}=0.8354$, Figure $4 B$ ). Similarly, the NET release process during irradiation was also suppressed by aprotinin (mean \pm SEM: $42.38 \% \pm 4.652 \%$ vs. $27.85 \% \pm 3.651 \%, \mathrm{P}=0.0205$, Figure $4 C$ ).

\section{Discussion}

Here, we found the novel regulatory mechanisms of aging neutrophils through LL37-NETs interactions to promote SVV, supplying basic and clinical implications. .

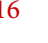



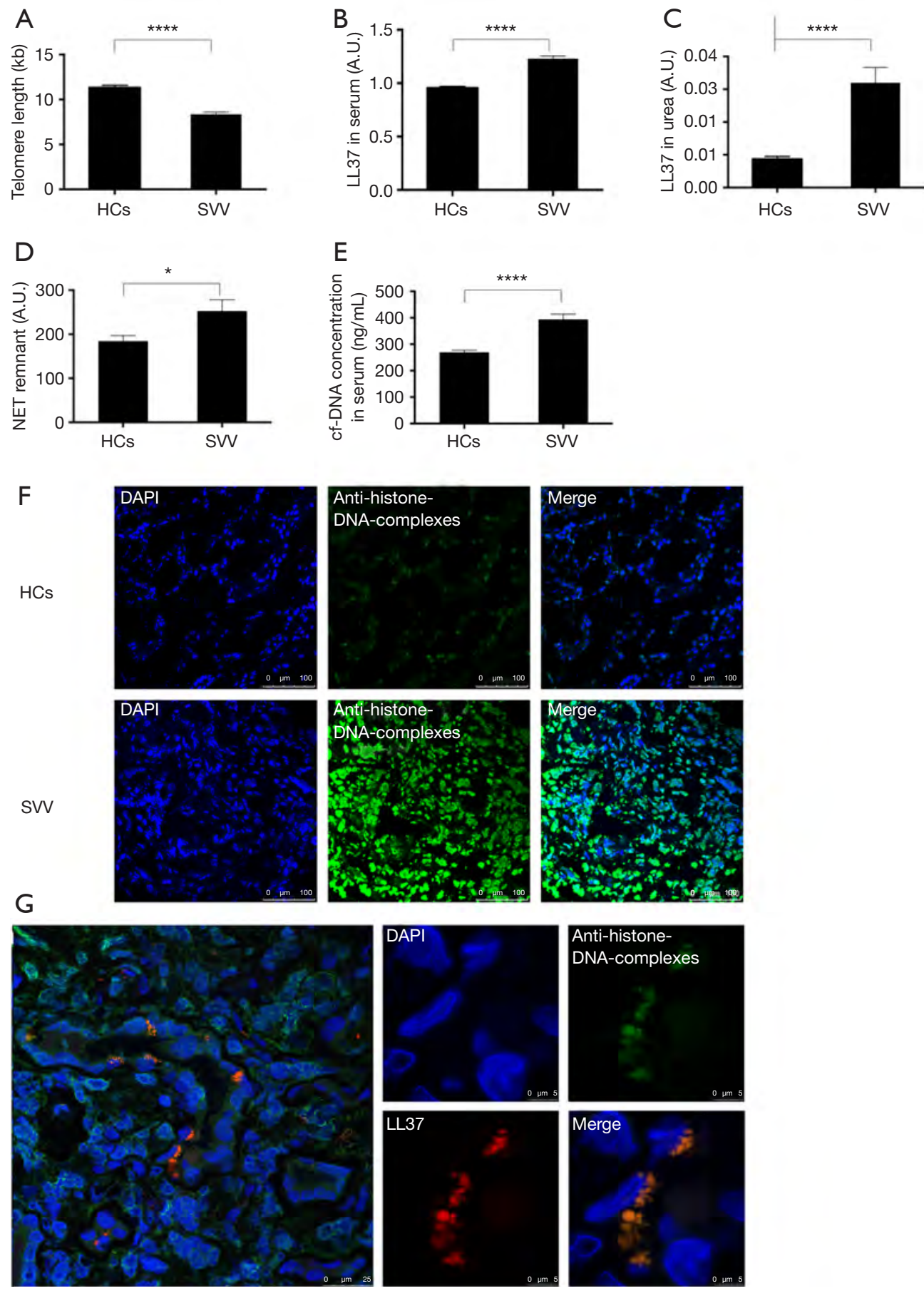

Figure 2 Higher levels of LL37 expression and NET release are determined in patients with SVV. (A) Telomere length of SVV and HCs by real-time Q-PCR; (B) levels of LL37 in serum from the disease group and healthy group; (C) levels of LL37 in urine from the disease group and healthy group; (D) levels of NET remnants in serum from patients with SVV or HCs; (E) levels of cf-DNA in serum from patients with SVV or HCs (A-E). N=70 for each group. The error bars of the graph represent the SEM. Unpaired Student's $t$-test was conducted to analyze the statistical significance of two independent experiments; (F) an Alexa 488-labeled antibody against H2A-H2B-DNA complexes was used to visualize NETs in green, and DAPI was used to stain the nuclei in blue. Pictures were merged to form an overlay image; (G) H2A-H2B-DNA complexes, LL37, and nuclei are stained green, red, and blue, respectively. Pictures were merged to form an overlay image. A.U., arbitrary unit. 

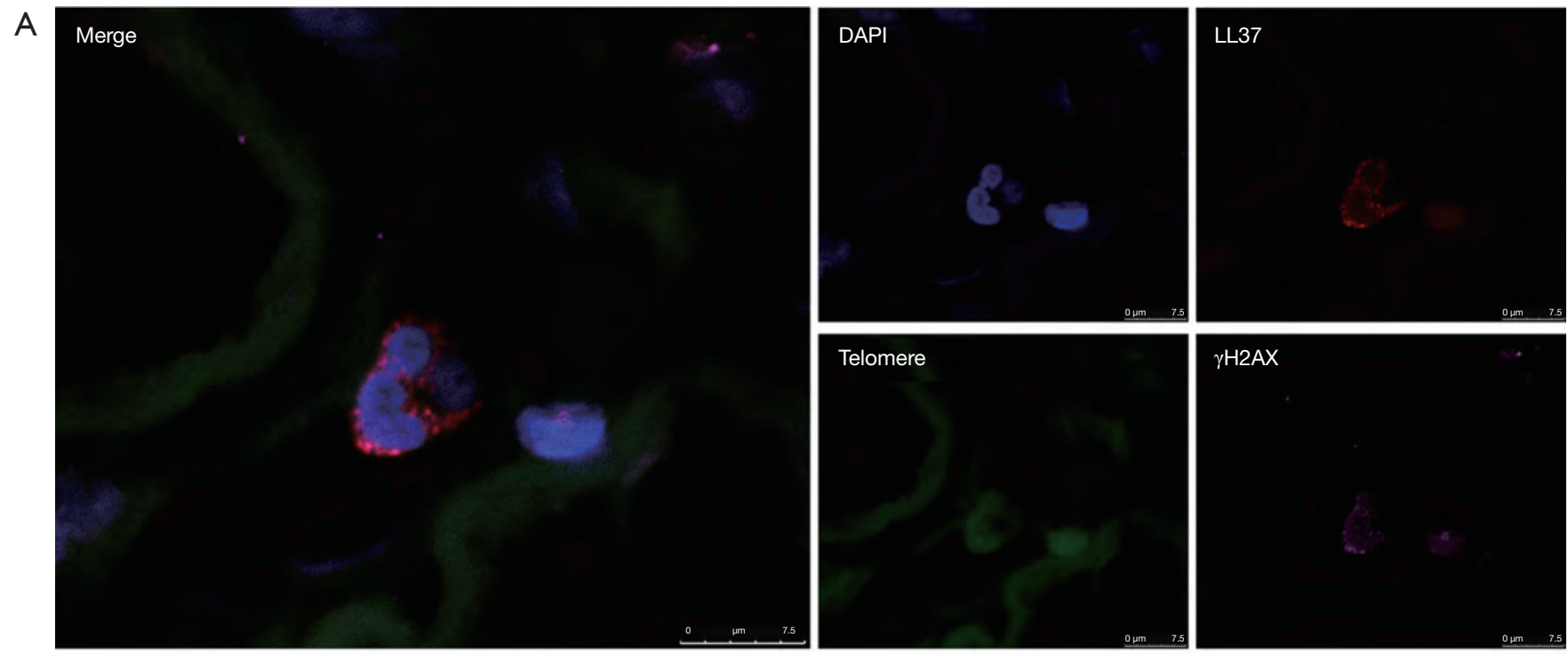

$\gamma \mathrm{H} 2 \mathrm{AX}$

B
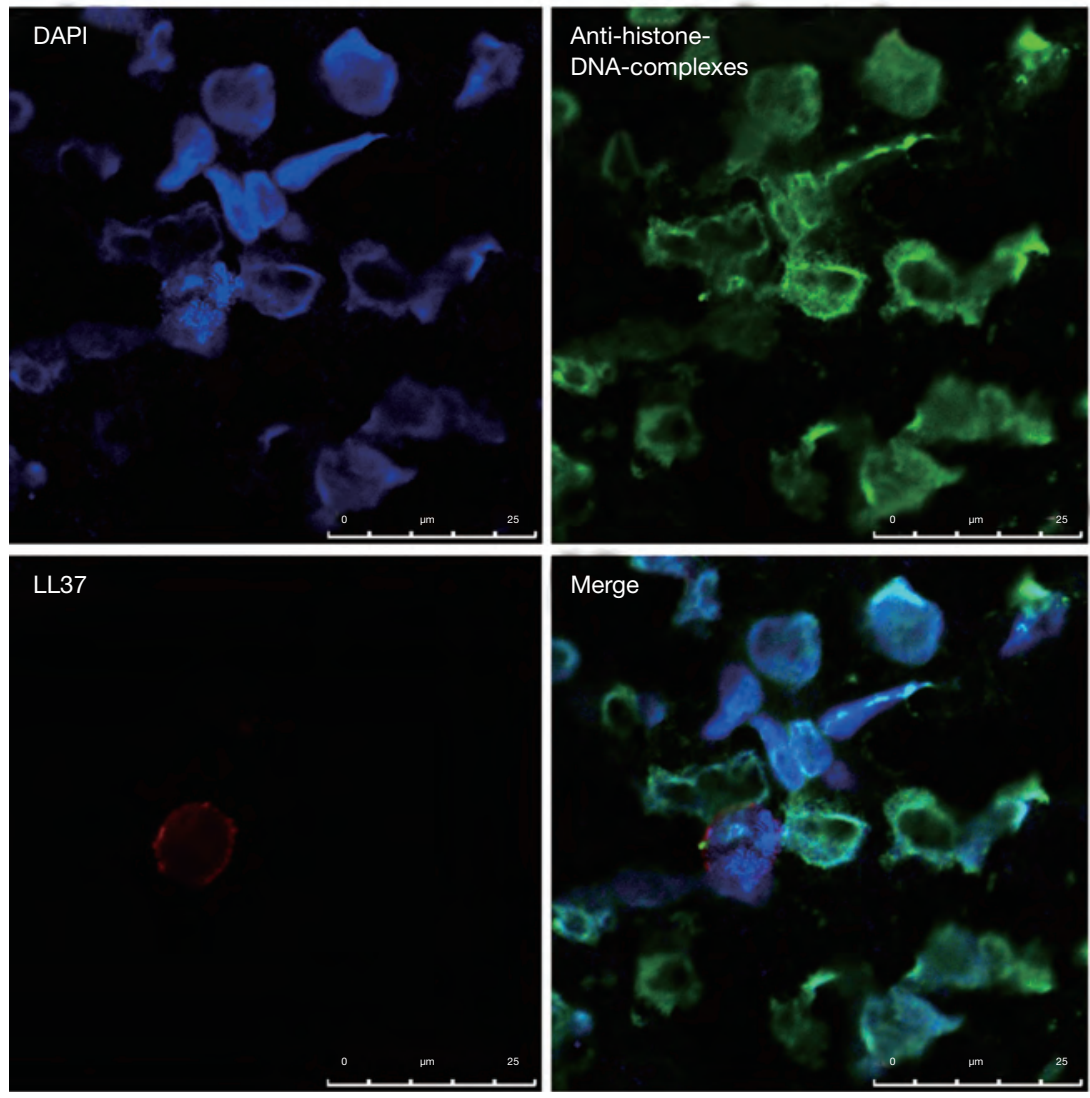

Figure 3 Immune aging of neutrophils and inflammatory medium release are determined in situ in individuals with SVV. (A) Neutrophils (polymorphic nuclear) infiltrated the kidney tissue from patients with SVV. Telomere dysfunction was found by the co-localization of $\gamma \mathrm{H} 2 \mathrm{AX}$ (purple) and telomeres (green) in the nucleus (DNA in blue). Immunostaining of LL37 showed perinuclear LL37 (red) in neutrophils that suffered DNA dysfunction; (B) H2A-H2B-DNA complexes, LL37, and nuclei are stained green, red, and blue, respectively. Pictures were merged to form an overlay image. LL37 assembled around the polymorphic nucleus in a background of NET deposition in the kidney tissue of SVV patients. 

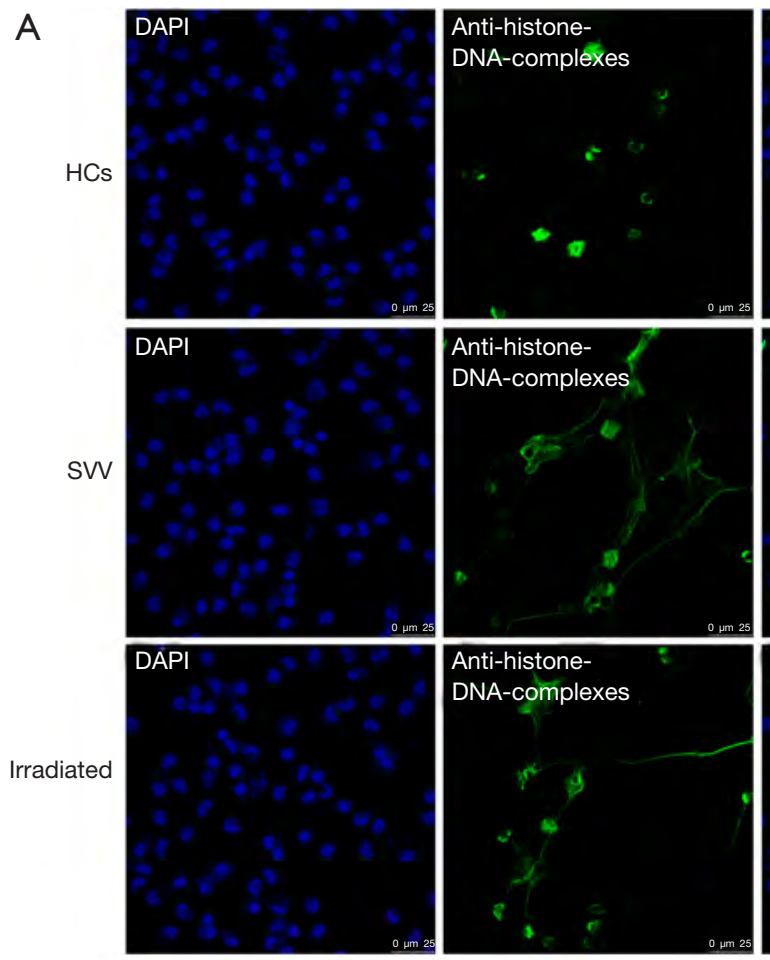

B

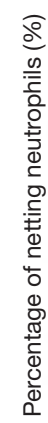

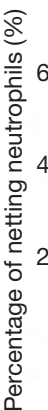

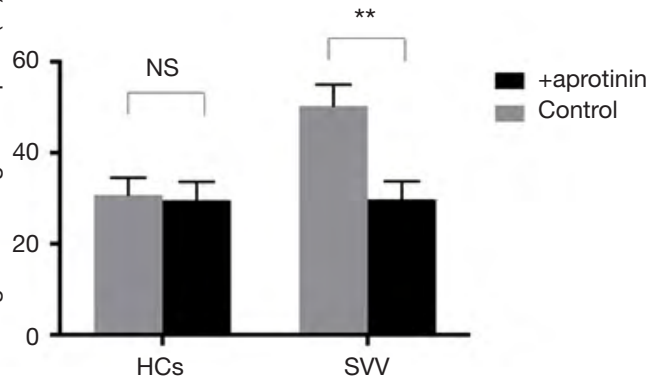

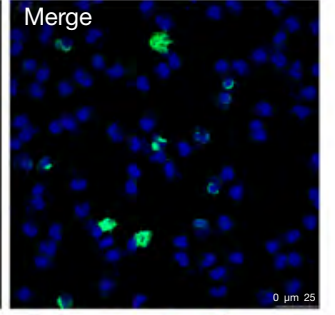

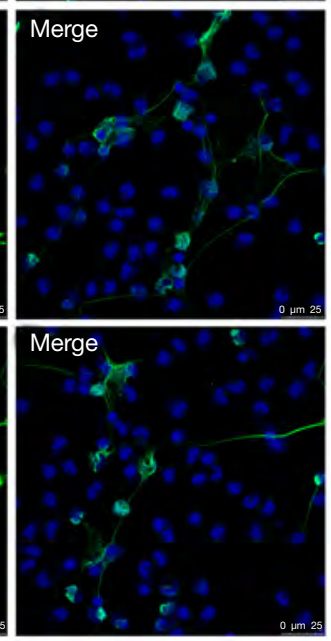

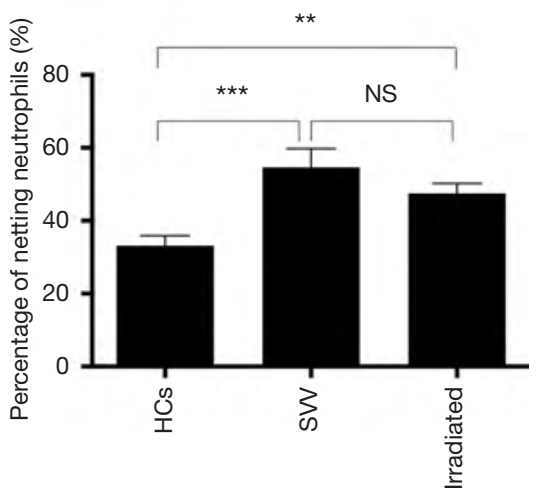

Figure 4 Human neutrophils from SVV produce more LL37-mediated-NETs. (A) The percentage of spontaneously netting neutrophils from healthy controls (HCs) and SVV patients and the percentage of netting neutrophils after irradiation; (B) degree of NET release of neutrophils from HCs and SVV in the presence or absence of aprotinin; (C) degree of NET release of neutrophils after irradiation in the presence or absence of aprotinin. The graph is a minimum of 15 images derived from 3 independent experiments. The error bars of the graph represent SEM. Unpaired Student's $t$-test was conducted to analyze the statistical significance of two independent experiments. ${ }^{* * *}$, $\mathrm{P}<0.001 ;{ }^{* *}, \mathrm{P}<0.01 ;{ }^{*}, \mathrm{P}<0.05 ; \mathrm{NS}$, not significant.

Increasingly evidence proves that NETs have a key role in tissue injury and dysfunction in systemic autoimmunity. The mechanism of how NETs affect end-organ tissues in SVV was well investigated, including interaction with myeloid dendritic cells (17) or endothelial cells (18), alternative complement pathway activation (19), and

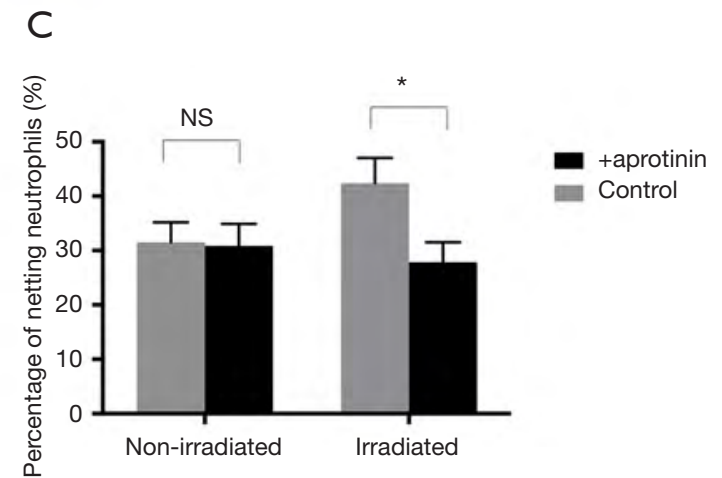

thrombin generation (20). However, the mechanisms mediating NET release need a better understanding. The present study supplies evidence for the first time depicting the NET response to telomere dysfunction and DNA damage (Figure $1 A, B)$. Interestingly, NETs were not associated with the normal aging of WT (Figure 1A). The 
observation that mouse telomeres had not shortened enough to show phenotypic changes during normal aging could partly explain this. Zhang et al. found aged neutrophils with high expression of CXCR4 and low expression of CD62L release excessive NETs, which strengthened our results that senescent neutrophils are overly-activated in circulation (21).

Earlier studies found that telomere-driven premature senescence showed in individuals with chronic activation of the immune system. Accelerated telomere loss was implicated in different leukocyte subpopulations as a common feature of autoimmune diseases (22-24). These studies mainly focused on monocyte and lymphocyte subpopulations (25-28). Evidence for telomere shortening of $T$ cells derived from patients with GPA, a subtype of SVV, was provided (29). The telomere erosion of neutrophils was discovered previously in SLE, however (30).

In the present study, we discovered for the first time that telomere erosion existed in neutrophils from individuals with SVV, with evidence of telomere shortening in neutrophils in circulation (Figure $2 A$ ) and DNA damage foci at dysfunctional telomeres in neutrophils infiltrating the renal tissue (Figure $2 F$ ). An increased renewal and replicative senescence due to chronic inflammation may accelerate telomere loss of leukocytes (31). Another factor may be the increased oxidative stress and oxidative DNA damage that cause telomere erosion, which has already been found in another autoimmune disease $(32,33)$.

Our earlier study showed that cramp, the homologous peptide of LL37 in mice, is a biomarker of aging and telomere dysfunction. Cramp was overexpressed in late generations of telomerase knockout mice (G4mTerc $\left.{ }^{-/}\right)$ and up-regulated in response to DNA damage after irradiation (11). Additionally, LL37 is a major component of NETs (31). After release by neutrophils, LL37 can interact with NETs and protect NETs from degradation by nucleases (9). Our present study speculated that LL37 might be associated with NETs release by aging neutrophils in SVV since the increased level of LL37 and NETs was identified in serum (Figure $2 B, D, E$ ) and kidney tissue (Figure $2 F, G$ ) from SVV patients. Zhang et al. strengthened our results, whose data suggested that higher levels of LL37 were observed in SVV patients, particularly those with crescentic formation (8).

Interestingly, we also found that LL37 surrounded the nucleus of neutrophils that suffered telomere dysfunction (Figure $3 A$ ) and produced a significant amount of NETs (Figure $3 B$ ) in the kidney tissue of SVV patients. This shows that LL37 may function as a bridge between telomere dysfunction and NET production of neutrophils. This may occur because neutrophils suffering from telomere dysfunction or DNA damage produce more LL37 (11). In our present study, we also found that inhibition of LL37 reduced the NETs released by neutrophils from SVV or after irradiation (Figure 4B,C). Telomere dysfunction taken together may promote SVV via the LL37-dependent NETs mechanism.

In conclusion, our study shows that the immune aging of neutrophils induced by DNA damage and telomere dysfunction could result in the over-release of NETs and the high expression of LL37. Consequently, NETs were deposited in the kidney tissue and caused persistent damage, finally affecting SVV disease progression. Notably, this study could not clarify the exact mechanism of LL37 regulation driven by telomere dysfunction. Further studies are needed to elucidate this process. Because many immunemediated pathologies share common immunological mechanisms biomarkers for SVV and other chronic inflammatory diseases that theoretically may be LL37 or NETs. Therefore, they may be established as antagonists in future therapies. Because many immune-mediated pathologies share common immunological mechanisms, LL37 or NETs may potentially be biomarkers for SVV and other chronic inflammatory diseases. Furthermore, their antagonists could be developed as potential therapies.

\section{Acknowledgments}

Funding: This work was supported by grants from the National Basic Research Program of China (2012CB517603), the National Natural Science Foundation of China $(81470938,81200546,81300619)$, the Major projects (2012C13G2010133), and Youth Fund Projects of the Zhejiang Science and Technology Department (LQ19H050005).

\section{Footnote}

Conflicts of Interest: The authors have no conflicts of interest to declare.

Ethical Statement: The authors are accountable for all aspects of the work in ensuring that questions related to the accuracy or integrity of any part of the work are appropriately investigated and resolved. The local Ethics Committee approved the study of the First Affiliated 
Hospital, School of Medicine, Zhejiang University (2016354 ), and written informed consent was obtained from all patients.

Open Access Statement: This is an Open Access article distributed in accordance with the Creative Commons Attribution-NonCommercial-NoDerivs 4.0 International License (CC BY-NC-ND 4.0), which permits the noncommercial replication and distribution of the article with the strict proviso that no changes or edits are made and the original work is properly cited (including links to both the formal publication through the relevant DOI and the license). See: https://creativecommons.org/licenses/by-nc-nd/4.0/.

\section{References}

1. Nakazawa D, Masuda S, Tomaru U, et al. Pathogenesis and therapeutic interventions for ANCA-associated vasculitis. Nat Rev Rheumatol 2019;15:91-101.

2. Geetha D, Jefferson JA. ANCA-Associated Vasculitis: Core Curriculum 2020. Am J Kidney Dis 2020;75:124-37.

3. Thieblemont N, Wright HL, Edwards SW, et al. Human neutrophils in auto-immunity. Semin Immunol 2016;28:159-73.

4. Schreiber A, Choi M. The role of neutrophils in causing antineutrophil cytoplasmic autoantibody-associated vasculitis. Curr Opin Hematol 2015;22:60-6.

5. Frangou E, Vassilopoulos D, Boletis J, et al. An emerging role of neutrophils and NETosis in chronic inflammation and fibrosis in systemic lupus erythematosus (SLE) and ANCA-associated vasculitides (AAV): Implications for the pathogenesis and treatment. Autoimmun Rev 2019;18:751-60.

6. Gupta S, Kaplan MJ. The role of neutrophils and NETosis in autoimmune and renal diseases. Nat Rev Nephrol 2016;12:402-13.

7. Papayannopoulos V. Neutrophil extracellular traps in immunity and disease. Nat Rev Immunol 2018;18:134-47.

8. Zhang Y, Shi W, Tang S, et al. The influence of cathelicidin LL37 in human anti-neutrophils cytoplasmic antibody (ANCA)-associated vasculitis. Arthritis Res Ther 2013;15:R161.

9. Neumann A, Vollger L, Berends ET, et al. Novel role of the antimicrobial peptide LL-37 in the protection of neutrophil extracellular traps against degradation by bacterial nucleases. J Innate Immun 2014;6:860-8.

10. Neumann A, Berends ET, Nerlich A, et al. The antimicrobial peptide LL-37 facilitates the formation of neutrophil extracellular traps. Biochem J 2014;464:3-11. 513

11. Jiang H, Schiffer E, Song Z, et al. Proteins induced 514 by telomere dysfunction and DNA damage represent 515 biomarkers of human aging and disease. Proc Natl Acad 516 Sci U S A 2008;105:11299-304.

12. Jennette JC, Falk RJ, Bacon PA, et al. 2012 revised International Chapel Hill Consensus Conference Nomenclature of Vasculitides. Arthritis Rheum 2013;65:1-11.

13. Cawthon RM. Telomere measurement by quantitative PCR. Nucleic Acids Res 2002;30:e47.

14. Söderberg D, Kurz T, Motamedi A, et al. Increased levels of neutrophil extracellular trap remnants in the circulation of patients with small vessel vasculitis, but an inverse correlation to anti-neutrophil cytoplasmic antibodies during remission. Rheumatology (Oxford) 2015;54:2085-94.

15. Rudolph KL, Chang S, Lee HW, et al. Longevity, stress response, and cancer in aging telomerase-deficient mice. Cell 1999;96:701-12.

16. Yamasaki K, Schauber J, Coda A, et al. Kallikreinmediated proteolysis regulates the antimicrobial effects of cathelicidins in skin. FASEB J 2006;20:2068-80.

17. Sangaletti S, Tripodo C, Chiodoni C, et al. Neutrophil extracellular traps mediate transfer of cytoplasmic neutrophil antigens to myeloid dendritic cells toward ANCA induction and associated autoimmunity. Blood 2012;120:3007-18.

18. Schreiber A, Rousselle A, Becker JU, et al. Necroptosis controls NET generation and mediates complement activation, endothelial damage, and autoimmune vasculitis. Proc Natl Acad Sci U S A 2017;114:E9618-25.

19. Huang YM, Wang H, Wang C, et al. Promotion of hypercoagulability in antineutrophil cytoplasmic antibodyassociated vasculitis by $\mathrm{C} 5 \mathrm{a}$-induced tissue factorexpressing microparticles and neutrophil extracellular traps. Arthritis Rheumatol 2015;67:2780-90.

20. Kambas K, Chrysanthopoulou A, Vassilopoulos D, et al. Tissue factor expression in neutrophil extracellular traps and neutrophil derived microparticles in antineutrophil cytoplasmic antibody associated vasculitis may promote thromboinflammation and the thrombophilic state associated with the disease. Ann Rheum Dis 2014;73:1854-63.

21. Zhang D, Chen G, Manwani D, et al. Neutrophil ageing is regulated by the microbiome. Nature 2015;525:528-32.

22. Costenbader KH, Prescott J, Zee RY, et al. Immunosenescence and rheumatoid arthritis: does telomere shortening predict impending disease? 
Autoimmun Rev 2011;10:569-73.

23. Coussens E, Grine L, Bostoen J, et al. Analysis of telomere length as predictive marker in psoriasis for comorbidities. Exp Dermatol 2016;25:388-90.

24. van den Hoogen LL, Sims GP, van Roon JA, et al. Aging and Systemic Lupus Erythematosus - Immunosenescence and Beyond. Curr Aging Sci 2015;8:158-77.

25. Fessler J, Raicht A, Husic R, et al. Premature senescence of T-cell subsets in axial spondyloarthritis. Ann Rheum Dis 2016;75:748-54.

26. Gounder SS, Abdullah BJJ, Radzuanb N, et al. Effect of Aging on NK Cell Population and Their Proliferation at Ex Vivo Culture Condition. Anal Cell Pathol (Amst) 2018;2018:7871814.

27. Invernizzi P, Bernuzzi F, Lleo A, et al. Telomere dysfunction in peripheral blood mononuclear cells from patients with primary biliary cirrhosis. Dig Liver Dis 2014;46:363-8.

28. Beier F, Balabanov S, Amberger CC, et al. Telomere length analysis in monocytes and lymphocytes from patients with systemic lupus erythematosus using multi-color flowFISH. Lupus 2007;16:955-62.

Cite this article as: Lu Y, Jiang H, Li B, Cao L, Shen Q, Yi W, Ju Z, Chen L, Han F, Appelgren D, Segelmark M, de Buhr N, von Köckritz-Blickwede M, Chen J. Telomere dysfunction promotes small vessel vasculitis via the LL37-NETs-dependent mechanism. Ann Transl Med 2020;8(6):357. doi: 10.21037/ atm.2020.02.130
29. Vogt S, Iking-Konert C, Hug F, et al. Shortening of 578 telomeres: Evidence for replicative senescence of T cells 579 derived from patients with Wegener's granulomatosis. $\quad 580$ Kidney Int 2003;63:2144-51.

30. $\mathrm{Wu} \mathrm{CH}$, Hsieh SC, Li KJ, et al. Premature telomere shortening in polymorphonuclear neutrophils from patients with systemic lupus erythematosus is related to the lupus disease activity. Lupus 2007;16:265-72.

31. Kordinas V, Ioannidis A, Chatzipanagiotou S. The Telomere/Telomerase System in Chronic Inflammatory Diseases. Cause or Effect? Genes (Basel) 2016. doi: 10.3390/genes7090060.

32. Tsai CY, Shen CY, Liao HT, et al. Molecular and Cellular Bases of Immunosenescence, Inflammation, and Cardiovascular Complications Mimicking "Inflammaging" in Patients with Systemic Lupus Erythematosus. Int J Mol Sci 2019. doi: 10.3390/ijms20163878.

33. Guan JZ, Guan WP, Maeda T, et al. Patients with multiple sclerosis show increased oxidative stress markers and somatic telomere length shortening. Mol Cell Biochem 2015;400:183-7. 